\title{
Disponibilidad de residuos forestales y su potencial para la generación de energía en los bosques templados de El Salto, Durango
}

\section{Availability of forest residues and potential for energy production in temperate forests in EI Salto, Durango}

\author{
Juan Martín Villela-Suárez!, Oscar Alberto Aguirre-Calderón², Eduardo Javier Treviño-Garza² y \\ Benedicto Vargas-Larreta ${ }^{3 *}$
}

Universidad Autónoma de Nuevo León. Programa de Doctorado de la Facultad de Ciencias Forestales. Linares, Nuevo León, México.

* Autor de correspondencia.

bvargas@itelsalto.edu.mx
2 Universidad Autónoma de Nuevo León. Facultad de Ciencias Forestales. Linares, Nuevo León, México.
* Instituto Tecnológico de El Salto. El Salto, P. N., Durango, México.

\section{RESUMEN}

Los residuos de la cosecha forestal son una fuente de energía renovable que no ha sido considerada todavía a gran escala en México y para su estimación se requiere de evaluaciones cada vez más precisas. En este estudio se desarrollaron ecuaciones para estimar el volumen de residuos forestales y se cuantificó la disponibilidad de biomasa y su potencial para generar energía eléctrica y reducir emisiones de $\mathrm{CO}_{2}$ en la región de El Salto, Durango. La metodología se dividió en tres fases: i) se obtuvo el volumen total autorizado de los programas de manejo forestal maderable de 20 ejidos de la región; ii) se derribaron y cubicaron 2156 árboles en las áreas de corta de los 20 ejidos para generar ecuaciones de volumen de residuos a escala de árbol individual; y iii) el volumen de residuos fue convertido a toneladas de biomasa empleando los valores de densidad de la madera para posteriormente transformar este valor a unidades de energía eléctrica. Dado que las especies estudiadas constituyen bosques mezclados, manejados con el mismo tratamiento silvícola y edad de rotación, se evaluaron las diferencias entre especies en las ecuaciones de volumen de residuos, utilizando el método de la suma adicional de cuadrados no lineales. Los parámetros de las ecuaciones de volumen de residuos fueron significativamente diferentes entre la mayoría de las especies. La cantidad de residuos a escala de árbol fue mayor en las especies del género Quercus, mientras que la cantidad de residuos forestales disponibles por ciclo de corta fue mayor en las especies del género Pinus. Alrededor de $31000 \mathrm{Mg}$ año-1 de residuos forestales pueden utilizarse en la región para generar aproximadamente $65.6 \mathrm{GW} \mathrm{h}$ año ${ }^{-1}$, considerando el uso de una tecnología con una eficiencia mínima de $40 \%$.

PALABRAS CLAVE: bioenergía, biomasa, ejido, $\mathrm{kW}$ h, programa de manejo.

\section{ABSTRACT}

Forest harvest residues are a source of renewable energyfor which their use has not yet been considered on a large scale in Mexico and for whose quantification, increasingly accurate assessments are required. In this study equations were developed to estimate the volume of forest residues in order to assess the regional availability of logging residues and their potential for electricity generation and $\mathrm{CO}_{2}$ emission displacement in the forest region El Salto, Durango. The methodology consisted of three steps: i) the forest management programs of 20 ejidos were revised to calculate the available total volume; (ii) 2156 trees from the harvest areas were felled and its volume estimated to develop tree-level residues equations; and (iii) the volume of waste was converted to tons of biomass using the wood density values each group species, and then this value was transformed to units of electrical energy. Due to these species often constitute mixed 
stands with the same silvicultural treatments and rotation age, differences among species in the volume residues equations were examined using the nonlinear extra sum of squares method. The model parameters were significantly different among species. The amount of forest residues at the tree scale was higher in the Quercus species, while the amount of forest residues was higher, per cut cycle, in Pinus species. Around $31000 \mathrm{Mg}$ year ${ }^{-1}$ of forest residues can be used to generate approximately $65.6 \mathrm{GW}_{\text {year }}^{-1}$, considering the use of a technology with a minimum efficiency of $40 \%$.

KEYWORDS: bioenergy, biomass, ejido, $\mathrm{kW}$ h, forest management program.

\section{INTRODUCCIÓN}

La utilización de biomasa forestal con fines bioenergéticos ha incrementado sustancialmente en los últimos años en muchas partes del mundo para convertirse en uno de los temas que reciben más atención de científicos, tomadores de decisiones y grupos de interés del sector energético en el contexto de las energías renovables (Ferranti, 2014). A pesar de sus bondades, la biomasa en general (no solo la forestal) no es considerada como la única alternativa para solucionar el problema de dependencia de los combustibles fósiles, por lo que su uso se ha combinado con otros tipos de tecnología limpia, como la hidroeléctrica, la solar o la geotérmica. De igual forma, la biomasa es la materia prima para otros tipos de energía renovable, como los biocombustibles (bioetanol, biodiesel) generados a partir de leña, residuos forestales, carbón vegetal, desechos agrícolas como la paja y el bagazo, así como algas y otros sólidos (Hamelinck, Van Hooijdonk y Faaij, 2005; Hammerschlag, 2006; Pérez-Verdin, Návar-Cháidez, Grebner y SotoÁlvarez, 2012). No obstante, la biomasa forestal, como fuente de energía renovable, es uno de los pilares de las políticas internacionales en la lucha por alcanzar los objetivos de reducción de gases de efecto invernadero (GEI). La idea de que la energía producida por la madera reduce las emisiones de GEI se basa en el supuesto de una neutralidad de carbono (Ferranti, 2014), que se asume real si se considera que la biomasa extraída del bosque y consumida en un proceso de generación de energía, es reemplazada en el largo plazo por nueva biomasa resultado del crecimiento del bosque, la cual reabsorbe el carbono emitido por el proceso de generación.

El watt (W) es una unidad de energía, por lo que un watthora es la energía necesaria para mantener una potencia constante de un watt durante una hora. Desde el punto de vista del consumo de energía, el kilowatthora $(\mathrm{kW} \mathrm{h})$ y megawatthora (MW h) son empleados más frecuentemente. El kilowatthora equivale a 1000 watthoras y se usa generalmente para la facturación del consumo eléctrico domiciliario, mientras que el MW h (1000 kW h), suele emplearse para medir el consumo de conglomerados urbanos. Por ejemplo, cuando se expresa el valor $100 \mathrm{~W} \mathrm{~h}$ se entiende indistintamente que se trata de la energía necesaria para mantener encendida una bombilla de $100 \mathrm{~W}$ durante una hora, o bien una pequeña luz de apenas $1 \mathrm{~W}$ durante 100 horas. Múltiplos aún más grandes como el gigawatt hora (GW h), el terawatthora (TW h) o el kilowattaño $(\mathrm{kW}$ año-1 $)$, son utilizados para referirse a las energías producidas por las centrales eléctricas durante un cierto período (Wright, Boundy, Perlack, Davis y Saulsbury, 2006).

El uso de la biomasa vegetal para producir electricidad creció en promedio 13 TW h año-1 del año 2000 al 2008, por lo cual en la actualidad existen muchos países productores de electricidad a partir de biomasa, siendo Estados Unidos el de mayor participación (26\%), seguido de Alemania (15\%), Brasil y Japón (ambos con 7\%) (Evans, Strezov y Evans, 2010). Se espera que la participación de la biomasa para la generación eléctrica mundial aumente 175\% en el año 2030 (Organización de las Naciones Unidas para la Alimentación y la Agricultura [FAO], 2010).

En los bosques de la región forestal de El Salto, Durango, una parte significativa de la biomasa forestal (ramas y puntas de los árboles), por lo general no se recupera durante el proceso de cosecha, ya que no se puede convertir fácilmente en productos utilizables como la madera aserrada, y son triturados y esparcidos o 
acomodados formando líneas sobre el terreno en la misma área de corta. Parte de estos residuos, principalmente de encino, son utilizados por los pobladores locales como leña para combustible en sus hogares. Al convertir los residuos en trozos pequeños, es posible su utilización como combustible y cuando se añaden los residuos de los aserraderos, el volumen de combustible disponible puede ser suficiente para crear una valiosa fuente de materia prima para su transformación en energía renovable (Kennedy, 2008). Estudios realizados a escala global (Smeets y Faaij, 2007) y en países como Rumania (Scarlat, Blujdea y Dallemand, 2011), Estados Unidos (Gan y Smith, 2006), Finlandia (Peltola, Kilpelainen y Asikainen, 2011) y Noruega (Hauglin, Gobakken, Lien, Bollandsas y Naesset, 2012) son muestra del actual interés en el aprovechamiento de los residuos forestales como una fuente importante de bioenergía.

El dióxido de carbono equivalente $\left(\mathrm{CO}_{2} \mathrm{e}\right)$ es una unidad universal de medición utilizada para indicar la posibilidad de calentamiento global de cada uno de los gases con efecto invernadero. En ese sentido, el "potencial de calentamiento global" de los tres gases con efecto invernadero asociados con la silvicultura es el siguiente: dióxido de carbono, que persiste en la atmósfera entre 200 años y 450 años, es definido como un potencial 1 del calentamiento mundial; el metano, que persiste en la atmósfera entre 9 años y 15 años, tiene un potencial de calentamiento global 22 (22 veces la capacidad de calentamiento del dióxido de carbono); y el óxido nitroso, que persiste por unos 120 años, tiene un potencial de calentamiento global 310 (Kennedy, 2008).

Estudios recientes presentan escenarios en los que se examina a detalle el papel futuro de la bioenergía en México. El primero de ellos (Islas, Manzini y Masera, 2007), establece que la bioenergía podría representar 16\% del total de la energía consumida en el país para el 2030, y podría mitigar $18 \%$ de las emisiones de $\mathrm{CO}_{2}$ para ese año. Los detalles de este estudio se presentan en Masera et al. (2006). El segundo estudio evaluó el potencial para reducir emisiones de GEI en México en el período 2008-2030 (Johnson, Alatorre, Romo y Liu, 2009), en el cual se estimó que las emisiones del escenario base alcanzarán 1137 megatoneladas de $\mathrm{CO}_{2}$ equivalente $\left(\mathrm{MtCO}_{2} \mathrm{e}\right)$ en 2030, partiendo de $659 \mathrm{MtCO}_{2} \mathrm{e}$ en 2008. En contraste, se reducirán las emisiones en $477 \mathrm{MtCO}_{2} \mathrm{e}$ para 2030 con respecto a la línea base. En otras palabras, según este escenario, México podría estabilizar sus emisiones de GEI en el año 2030 con estas opciones, pero las aumentaría 72\% si no lo hace.

Pese a su importancia, en México aún existe poca información respecto a la cuantificación y disponibilidad de residuos derivados de la cosecha forestal, así como de su potencial para la generación de bioenergía.

\section{OBJETIVOS}

Desarrollar ecuaciones de volumen de residuos forestales en la región de El Salto, Durango, así como cuantificar la disponibilidad de biomasa y evaluar su potencial de generación de energía eléctrica y de reducción de emisiones de carbono.

\section{MATERIALES Y MÉTODOS}

\section{Área de estudio}

$\mathrm{El}$ área de estudio abarca una superficie bajo manejo de 123822 ha y se ubica en el suroeste del estado de Durango, en la región forestal de Pueblo Nuevo (Fig. 1). Se localiza dentro de la cadena de montañas de la Sierra Madre Occidental, la cual se considera una de las regiones con mayor biodiversidad de América del Norte y contiene aproximadamente dos terceras partes de las existencias volumétricas de México, 23 especies de pino y cerca de 200 especies de encino (World Wildlife Found y Hogan, 2013).

\section{Muestra}

Para estimar la proporción que representa el volumen de las puntas y ramas respecto al volumen total del árbol, se derribaron y seccionaron 2156 árboles de especies comerciales (Tabla 1). La cubicación de los árboles se realizó por secciones usando la fórmula de Smalian; la punta se cubicó como un cono. El volumen de las ramas se calculó siguiendo el mismo procedimiento (por secciones). 


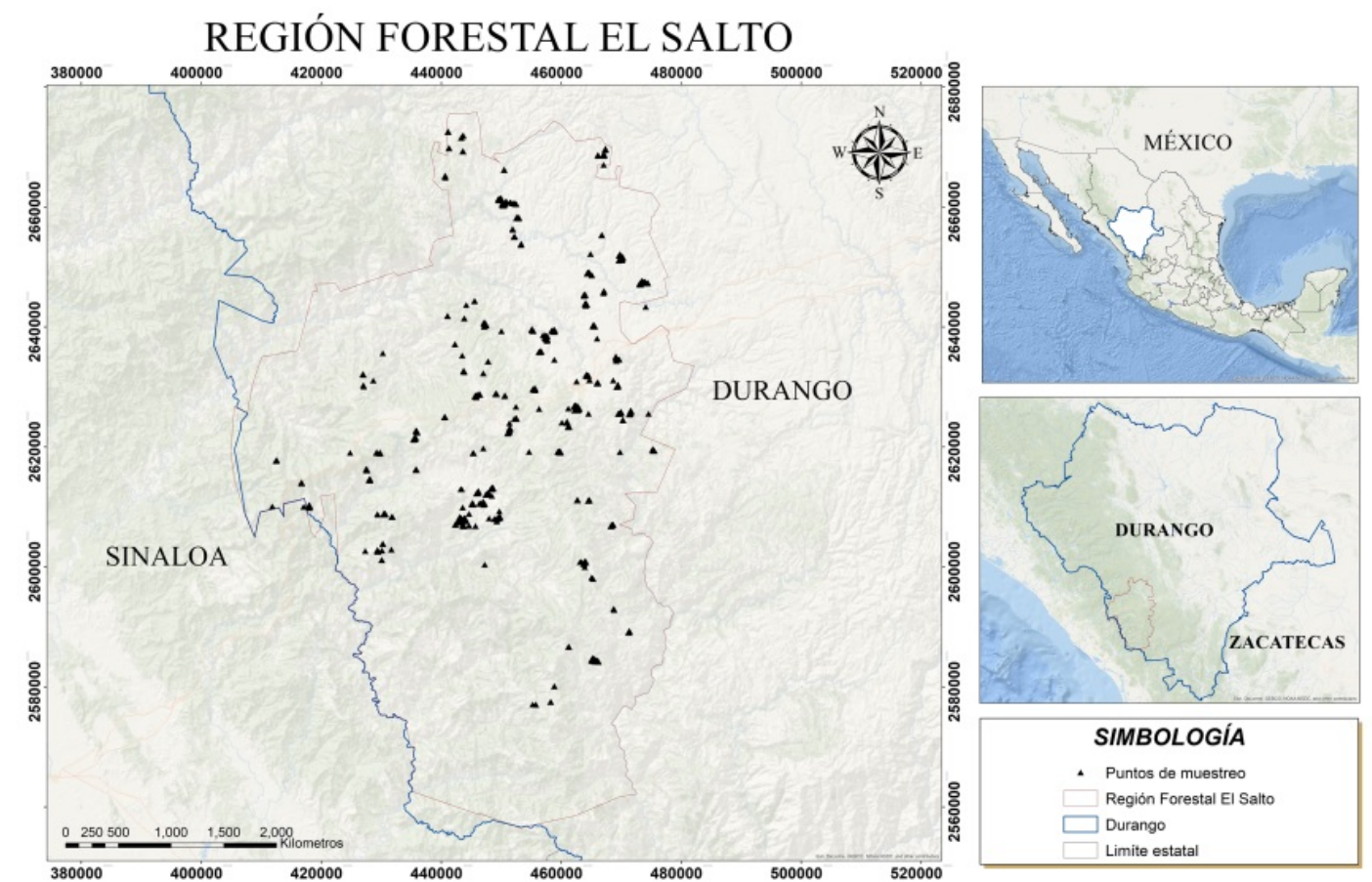

FIGURA 1. Ubicación del área de estudio en el contexto nacional y estatal (en negro los puntos de muestreo).

TABLA 1. Distribución de la muestra por especie.

\begin{tabular}{llcc}
\hline Código & Especie & $\begin{array}{c}\text { Intervalo diamétrico } \\
(\mathrm{cm})\end{array}$ & No. de árboles \\
\hline 1 & Pinus cooperi & $10.0-61.6$ & 167 \\
2 & Pinus durangensis & $9.2-68.6$ & 194 \\
3 & Pinus engelmannii & $9.5-52.5$ & 166 \\
4 & Pinus leiophylla & $9.5-58.5$ & 174 \\
5 & Pinus herrerae & $9.8-59.5$ & 148 \\
6 & Pinus teocote & $8.6-63.3$ & 177 \\
7 & Pinus lumholtzii & $8.5-45.7$ & 107 \\
8 & Pinus ayacahuite & $8.3-54.8$ & 106 \\
9 & Pinus oocarpa & $9.0-46.6$ & 160 \\
10 & Pinus douglasiana & $9.5-43.2$ & 217 \\
11 & Pinus michoacana & $9.1-60.3$ & 141 \\
12 & Pinus maximinoi & $10.0-45.3$ & 87 \\
170 & Quercus spp & $8.2-57.5$ & 164 \\
172 & Quercus durifolia & $9.5-56.5$ & 148 \\
\hline & Total & & 2156 \\
\hline
\end{tabular}


El volumen total del árbol se estimó sumando el volumen total del fuste (secciones + punta) y de las ramas. Debido al pequeño tamaño de muestra obtenido para $Q$. rugosa, $Q$. fulva, Q. sideroxyla y Q. crassifolia, estas especies se clasificaron como el grupo Quercus spp; para Quercus durifolia sí se tuvo el tamaño de muestra suficiente para ser considerada de manera independiente en el análisis. Los árboles fueron seleccionados mediante un muestreo dirigido para asegurarse de que estuvieran representadas todas las calidades de estación de la región, así como también la distribución de los árboles en términos de clases de diámetro y altura. A cada árbol se le midió el diámetro normal con corteza (D, en centímetros), la altura total ( $\mathrm{H}$, en metros), el diámetro de copa ( $\mathrm{C}$, en metros), el diámetro con corteza de cada troza ( $d$, en centímetros), la longitud de cada troza (l, en metros) y el diámetro con corteza para todas las ramas de más de $5 \mathrm{~cm}$ de diámetro en la base. Una vez seccionados los árboles, se desramaron y se procedió a la separación en sus diferentes fracciones de biomasa (fuste, puntas y ramas). Todos los componentes fueron pesados en campo con una báscula con resolución de $\pm 0.98 \mathrm{~N}(0.1 \mathrm{~kg})$, marca Torrey ${ }^{\circledR}$, modelo EQM 200-400 con capacidad de $9807 \mathrm{~N}$ (1 t); además, los discos de madera (rodajas) obtenidos de cada sección, así como una muestra de ramas $(19.6 \mathrm{~N} ; 2 \mathrm{~kg})$ obtenidas al azar de la copa del árbol, fueron pesados en verde y llevados a laboratorio para ser secados en una estufa marca Yamato, modelo DNE910, a una temperatura de 70 ${ }^{\circ} \mathrm{C}$ hasta peso constante. Esta información permitió calcular la relación volumen verde-peso seco.

\section{Cuantificación del volumen de residuos forestales}

El volumen de las trozas, así como el de las secciones de las ramas, se calculó mediante la fórmula de Smalian, mientras que el volumen de las puntas de los fustes y ramas fue calculado con la fórmula del cono; el diámetro en la base de las puntas osciló entre $5 \mathrm{~cm}$ y $10 \mathrm{~cm}$. Las ramas menores de $5 \mathrm{~cm}$ de diámetro en la base, las hojas o acículas y las raíces no se incluyeron en la evaluación, ya que fueron consideradas como criterio de restricción ecológica para garantizar la incorporación de nutrientes al suelo
(Borjesson, 2000). Los tocones tampoco fueron incluidos, ya que en la región estos no se consideran residuo recuperable de la cosecha. Para calcular el volumen de residuos disponible a escala de árbol, el volumen de la punta $\left(v_{\text {punta, }}, \mathrm{m}^{3}\right)$ se restó del volumen del fuste $\left(v_{\mathrm{f}}, \mathrm{m}^{3}\right)$; una vez conocido el volumen de ramas $\left(v_{\text {ramas }}, \mathrm{m}^{3}\right)$ se calculó el factor que estima los residuos, es decir, el volumen de las ramas más el de la punta $\left(v_{\text {rp }}, \mathrm{m}^{3}\right)$ respecto al volumen total $\left(v_{\text {total }}=v_{\mathrm{f}}+v_{\text {ramas }}\right)$ mediante la relación:

$$
v_{r p}=\left(v_{\text {ramas }}+v_{\text {punta }}\right) / v_{\text {total }}
$$

\section{Modelación del volumen de residuos}

Para el ajuste de las ecuaciones del volumen de residuos a escala de árbol individual, se emplearon tres enfoques: 1) ajustar la ecuación de forma independiente a cada una de las especies objeto de estudio, para lo cual el volumen de residuos disponible $\left(v_{\mathrm{r}}=v_{\text {ramas }}+v_{\text {punta }}\right)$ a escala de árbol individual fue modelado utilizando la ecuación 2 , que relaciona el volumen de residuos con el diámetro a la altura de pecho y el diámetro de la copa del árbol.

$$
v r=a_{0} \cdot \mathrm{D}^{a_{1}} \cdot \mathrm{C}^{a_{2}}+e
$$

donde:

$v_{\mathrm{r}}=$ volumen de residuos $\left(\mathrm{m}^{3}\right)$

$\mathrm{D}=$ diámetro normal $(\mathrm{cm})$

$\mathrm{C}=$ diámetro de copa $(\mathrm{m})$

$a_{\mathrm{i}}=$ parámetros del modelo

$e=$ error con distribución normal

2) agrupar por pares aquellas especies cuyos modelos no resultaron significativamente mejores en el ajuste individual y ajustar un modelo único que agrupara ambas especies, donde la hipótesis a probar fue que no existe ganancia en la disminución de los errores al utilizar el modelo único o los modelos individuales por especie; y 3) agrupar las especies por género y ajustar una única ecuación para cada grupo. El tercer enfoque tiene la ventaja de que no se requiere distinguir una especie de otra en campo para aplicar una ecuación determinada, aunque podría tener el inconve- 
niente de que los errores pueden ser mayores, en algunos casos, que los obtenidos con una ecuación específica para cada especie (primer enfoque) o par de especies (segundo enfoque). El tercer enfoque se considera una solución intermedia entre los dos primeros, puesto que, en el caso de poder agrupar especies, se reduce el número de ecuaciones y evita la diferenciación de especies en campo, sin que la pérdida de bondad de ajuste de las ecuaciones sea estadísticamente significativa.

Para evaluar si la ecuación de volumen de residuos (ec. 2) difiere entre especies de un mismo género, se ajustaron dos modelos diferentes: un modelo denominado reducido, en el que los datos del par de especies agrupadas (especies $i$, ) se unen y se ajusta la ecuación 2 a todo el conjunto de datos; y un modelo denominado completo en el que cada parámetro de la ecuación 2 se expande empleando una variable dicotómica dummy para diferenciar entre especies; de esta manera se obtuvo la ecuación 3:

$$
v_{\mathrm{r}}=\left(a_{0 \mathrm{i}}+a_{0 \mathrm{j}} I_{\mathrm{j}}\right) D^{\left(a_{1 \mathrm{i}}+a_{1 \mathrm{j}} I_{\mathrm{j}}\right)} C^{\left(a_{2 \mathrm{i}}+a_{2 \mathrm{j}} \mathrm{I}_{\mathrm{j}}\right)}
$$

donde $a_{\mathrm{ij}}$ son parámetros del modelo e $I_{\mathrm{j}}$ es una variable dicotómica que toma el valor 1 para la especie de referencia $i$ (que será la primera en cada combinación de pares de especies) y el valor 0 para la especie $j$ (la segunda en cada par de especies). El modelo reducido correspondió al mismo conjunto de parámetros para cada par de especies comparadas, mientras que el modelo completo correspondió a diferentes conjuntos de parámetros para cada especie. La comparación entre el modelo reducido y el modelo completo para cada par de especies analizado se realizó mediante el método de la suma adicional de cuadrados no lineales (Bates y Watts, 1988) cuya prueba estadística $F^{*}$ se define como:

$$
F^{*}=\frac{[\operatorname{SSE}(R)-S S E(C)] /\left[d f_{\mathrm{R}}-d f_{\mathrm{C}}\right]}{\left[\operatorname{SSE}(C) / d f_{\mathrm{C}}\right]}
$$

donde $F^{*}$ sigue una distribución $F$ de Snedecor; $\operatorname{SSE}(R)$ es la suma de cuadrados del error del modelo reducido; SSE (C) es la suma de cuadrados del error del modelo completo $\mathrm{y} d f_{\mathrm{R}} \mathrm{y} d f_{\mathrm{C}}$ son los grados de libertad del error en el modelo reducido y en el modelo completo, respectivamente.

El valor de la prueba de $F^{*}$ obtenido con la ecuación 4 se comparó con el valor tabulado de $F \operatorname{con} \alpha=0.05$; si $F^{*}$ $>F$ tabulada $\left(1-\alpha ; d f_{\mathrm{R}}-d f_{\mathrm{C}}, d f_{\mathrm{C}}\right)$ o si la probabilidad asociada $(P>F$ tabulada $)<\alpha$, se rechaza la hipótesis nula $\left(\mathrm{H}_{0}\right.$ : no existe diferencia entre especies), lo cual implica que los parámetros del modelo de regresión difieren entre especies por lo que se requiere el uso de una ecuación específica para la predicción del volumen de residuos de las especies comparadas (Weisberg, 1980; Draper y Smith, 1981; Montgomery y Peck, 1982). En caso contrario, se aceptaría el uso del modelo reducido (mismo conjunto de parámetros) para las especies agrupadas. El modelo completo (ec. 4) de cada una de las posibles combinaciones de pares de especies de pino se comparó con su correspondiente modelo reducido usando la prueba de $F^{*}$. Solamente en los casos en que se obtuvo un valor de $F(P$ $>0.05)$ se consideró que la ecuación de volumen de residuos para esas dos especies era similar y, por lo tanto, dichas especies se combinaron.

Análisis preliminares de los residuos mostraron un problema de heterocedasticidad asociado al uso de modelos alométricos para estimar el volumen de residuos, lo que supone una violación de las hipótesis de partida del ajuste por mínimos cuadrados. Para corregirlo, el ajuste de los modelos se llevó a cabo mediante regresión ponderada, de modo que a cada observación se le asignó un peso igual a la inversa de su varianza $\sigma_{i}^{2}$. Aunque esta varianza es desconocida, se considera que la varianza del error de la $i$ ésima observación puede ser modelizada como una función potencial de las variables independientes del modelo empleado, en este caso $D$ y $C$, por tanto, $\sigma_{i}^{2}=\left(D^{2} C\right)^{k}$ (Harvey, 1976). El valor del término potencial k se optimizó para obtener el gráfico de distribución de residuos estudentizados más homogéneo. Los parámetros fueron estimados con el procedimiento PROC MODEL del paquete estadístico SAS/ETSTM (Statistical Analysis System [SAS], 2011). 


\section{Análisis estadístico}

La bondad de ajuste de los modelos se midió a través del análisis de los residuales y mediante el empleo de dos estadísticos: el coeficiente de determinación ajustado $\left(R_{\text {adj }}^{2}\right)$ (ec. 5) y la raíz del error medio cuadrático (REMC) (ec. 6), así como la significancia de los parámetros a través del error estándar (EStd) y el valor de $t(\mathrm{pr}>|\mathrm{t}|)$.

$$
\begin{gathered}
\mathrm{R}_{a d j}^{2}=1-\frac{(n-1) \sum_{i=1}^{n}\left(y_{i}-\hat{y}_{i}\right)^{2}}{(n-p) \sum_{i=1}^{n}\left(y_{i}-\bar{y}\right)^{2}} \\
\mathrm{REMC}=\sqrt{\frac{\sum_{i=1}^{n}\left(y_{i}-\hat{y}_{i}\right)^{2}}{n-p}}
\end{gathered}
$$

donde $y_{i}, \hat{y}_{i} \mathrm{e}^{\bar{y}}$ son los valores observados, predichos y promedio de la variable dependiente, $n$ es el número de observaciones y $p$ es el número de parámetros del modelo.

\section{Cuantificación del volumen de residuos disponibles a escala regional}

Con las ecuaciones desarrolladas se estimó el volumen de residuos disponibles a escala árbol y por especie, así como para el conjunto de árboles a escala subrodal, rodal y predio, calculando el volumen de residuos para el árbol tipo de cada categoría diamétrica y especie y multiplicando este valor por el número de árboles de dicha categoría. Las ecuaciones de volumen de residuos desarrolladas se aplicaron a los datos de los inventarios de los programas de manejo de los 20 ejidos de la región y se cuantificaron los residuos forestales disponibles en el área de estudio. Los bosques de la región se manejan con un sistema silvícola que se basa en los principios del manejo regular e irregular, bajo los lineamientos del Método de Desarrollo Silvícola (MDS), el cual consta de una corta de establecimiento, tres o cuatro cortas intermedias y una corta final, y del Método Mexicano de Ordenación de Bosques Irregulares (MMOBI), que se caracteriza por la aplicación de cortas selectivas a lo largo de la vida de la masa (Pro Floresta, 2008).

\section{Generación de electricidad y desplazamiento de carbono}

El siguiente paso fue transformar el volumen de residuos recuperables a electricidad potencialmente generable. Dicha conversión involucró, primero, la trasformación del volumen de residuos a peso de biomasa, para lo cual se empleó la ecuación $W_{\mathrm{r}}=d_{\mathrm{m}} \cdot v r$, donde $W_{\mathrm{r}}$ es la cantidad de residuos secos (biomasa, en $\mathrm{Mg}$ ), $d_{\mathrm{m}}$ es la densidad promedio de la madera y $v r$ es el volumen de residuos $\left(\mathrm{m}^{3}\right)$. Debido a la coexistencia de múltiples especies en los bosques del área de estudio, la densidad básica utilizada fue la señalada por Kennedy (2008), quien obtuvo un valor promedio ponderado de las especies más comunes (0.46 para el grupo de especies de Pinus y 0.64 para las especies de Quercus). La suma de la biomasa disponible por especie y por ejido fue luego convertida a cantidad de energía eléctrica generable, para lo cual se utilizó el valor mencionado por Gan y Smith (2006), quienes señalan que 1 tonelada de biomasa contiene una energía de 19.19 GJ, equivalente a $0.0053 \mathrm{GW}$ h.

Con base en las estimaciones de electricidad generada, se calculó la cantidad de emisiones de $\mathrm{CO}_{2}$ que sería posible desplazar al sustituir el proceso de generación de energía eléctrica actual por una tecnología de generación a partir de residuos de biomasa forestal. Para esto se utilizó el factor 0.54 publicado por Kennedy (2008), el cual indica que 1 tonelada de biomasa quemada en un sistema de generación equivale a 0.54 toneladas de $\mathrm{CO}_{2}$ equivalente $\left(\mathrm{tCO}_{2} \mathrm{e}\right)$ de reducción de emisiones. Se consideró que las emisiones netas de una planta de generación a partir de biomasa son iguales a cero (Evans et al., 2010).

\section{Resultados}

Modelo predictivo de residuos a escala de árbol individual

El intervalo de valores de residuos disponibles (biomasa) a escala de árbol individual osciló entre $0.003 \mathrm{~m}^{3}(1.39 \mathrm{~kg}$ ) 
para Pinus teocote y $0.44 \mathrm{~m}^{3}(202.21 \mathrm{~kg})$ para P. herrerae, en el grupo Pinus, y entre $0.025 \mathrm{~m}^{3}(1.52 \mathrm{~kg})$ y $0.485 \mathrm{~m}^{3}(310.3$ $\mathrm{kg}$ ) para Quercus durifolia dentro del grupo Quercus. En todos los casos, la cantidad de residuos incrementó marcadamente con el diámetro normal (Fig. 2). En la tabla 2 se muestran los estimadores de los parámetros, su error estándar y la significancia, así como los estadísticos de bondad de ajuste del modelo individual para cada especie (ec. 2).

Los parámetros fueron altamente significativos $(\alpha=$ $5 \%$ ), excepto el parámetro $a_{0}$ para las especies $1,2,4,5,9$ y 12. En todo caso, puesto que el modelo no tiene sentido sin este parámetro, se decidió mantenerlo. Se obtuvieron valores de $\mathrm{R}_{\text {adj }}^{2}$ relativamente bajos (por ejemplo 0.645 para la especie 9), lo cual se debe en parte a la gran dispersión que presenta el volumen de residuos para una misma categoría diamétrica por especie (Fig. 2). La ecuación de residuos propuesta requiere de información del diámetro de copa (C), una variable que es poco común medir en los inventarios forestales, y su inclusión en el modelo mejoró significativamente el ajuste, comparado con el modelo que solamente considera el diámetro normal, excepto en el caso de las especies 4, 6 y 9, en el que el parámetro asociado con el diámetro de copa no resultó significativo $(\alpha=5 \%)$ y fue descartado.

La cantidad del volumen total de residuos estimado varió considerablemente entre especies (Fig. 3), pero todas las estimaciones estuvieron dentro de los intervalos de valores observados de volumen de residuos.

\section{Comparación del volumen de residuos entre especies}

Los resultados del método de la suma adicional de cuadrados no lineales obtenidos en el ajuste de los modelos reducido y completo se muestran en la tabla 3. Se compararon 67 combinaciones de pares de especies, y en la mayoría de los casos (59), el modelo completo fue mejor que el modelo reducido, ya que los valores de $F^{*}$ resultaron altamente significativos $(P \leq 0.05)$; por lo tanto, para estas
59 comparaciones pareadas se rechazó la hipótesis nula de que un modelo único es adecuado para el par de especies analizado.

Como se mencionó, la hipótesis de partida es válida solamente para las especies de Quercus y los pares de especies de Pinus señalados anteriormente (resaltados en negrita en la tabla 3), por lo que se puede utilizar una ecuación única para estimar su volumen de residuos. En la tabla 4 se presentan los estimadores de los parámetros, sus errores estándar y los estadísticos de ajuste para el volumen de residuos forestales del modelo reducido para los casos en que se puede utilizar una misma ecuación para un par de especies.

\section{Estimación de residuos por grupo de especies}

El ajuste de una ecuación a escala de género (grupo Pinus y grupo Quercus), arrojó los resultados que se presentan en la tabla 5. En ambos casos los parámetros fueron altamente significativos y los estadísticos de bondad de ajuste satisfactorios; incluso el ajuste fue mejor que el obtenido al ajustar una ecuación individual para algunas especies de pino, como P. leiophylla (4), P. occarpa (9) y P. maximinoi (12), que presentaron valores de $0.72,0.64$ y 0.73 de $R_{\text {adj }}^{2}$, respectivamente (Tabla 2), menores al obtenido para el grupo Pinus (0.75) (Tabla 5). En el caso del grupo Quercus los resultados fueron similares.

\section{Estimación de residuos forestales a escala regional} Los modelos de residuos desarrollados se aplicaron a los datos de inventario de los ejidos para determinar la cantidad de biomasa disponible a escala regional. La cantidad de residuos recuperables en la región asciende a $60000 \mathrm{~m}^{3}$ año-1, que equivalen a $30940 \mathrm{Mg}$ año-1 de biomasa disponible para generación de energía eléctrica (Tablas 6 y 7). El volumen promedio de biomasa disponible por año fue mayor en las especies de Pinus, con $19067 \mathrm{Mg}$ año-1 (Tabla 6), mientras que para las especies de Quercus el valor promedio fue $11872 \mathrm{Mg}$ año-1 $^{-1}$ (Tabla 7). 

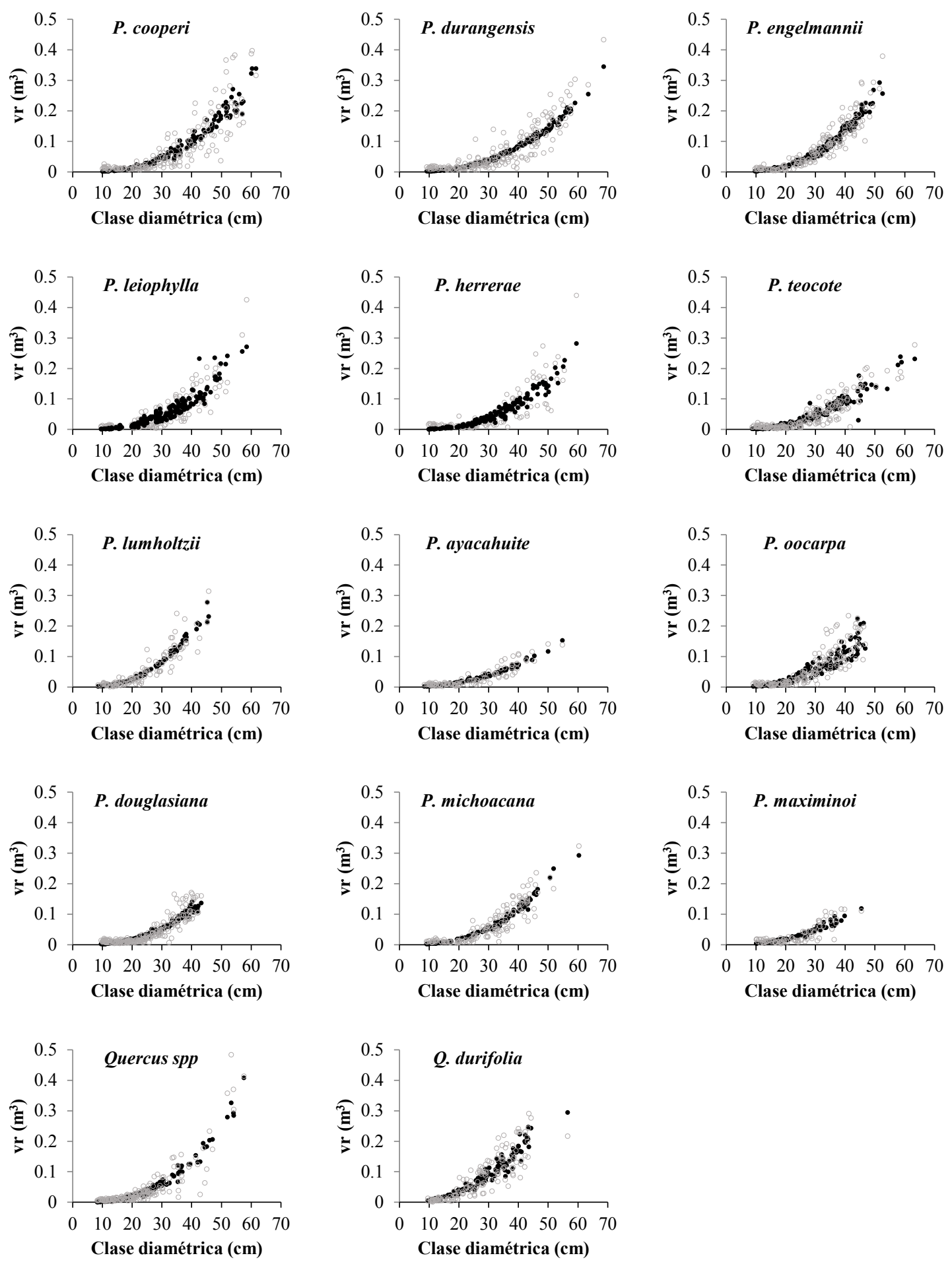

FIGURA 2. Volumen total de residuos $\left(\mathrm{m}^{3}\right)$ observado (círculos grises) y estimado (círculos negros) para cada especie. 
TABLA 2. Parámetros estimados y estadísticos de bondad de ajuste del modelo individual

\begin{tabular}{|c|c|c|c|c|c|}
\hline$S p$ & Parm & Estimador & EStd & $\operatorname{REMC}\left(\mathrm{m}^{3}\right)$ & $R^{2}$ adj \\
\hline \multirow[t]{3}{*}{1} & ao & 0.000009 & 0.000005 & 0.043 & 0.780 \\
\hline & $a_{1}$ & 2.136932 & 0.2803 & & \\
\hline & $a_{2}$ & 0.688671 & 0.2671 & & \\
\hline \multirow[t]{3}{*}{2} & ao & 0.00001 & 0.000005 & 0.030 & 0.836 \\
\hline & $a_{1}$ & 2.261934 & 0.2032 & & \\
\hline & $a_{2}$ & 0.340109 & 0.1771 & & \\
\hline \multirow[t]{3}{*}{3} & ao & 0.00001 & 0.000004 & 0.026 & 0.879 \\
\hline & $a_{1}$ & 2.303995 & 0.1966 & & \\
\hline & $a_{2}$ & 0.511378 & 0.1647 & & \\
\hline \multirow[t]{2}{*}{4} & ao & 0.000013 & 0.000009 & 0.031 & 0.729 \\
\hline & $a_{1}$ & 1.87904 & 0.1892 & & \\
\hline \multirow[t]{3}{*}{5} & ao & 0.000011 & 0.000009 & 0.031 & 0.773 \\
\hline & $a_{1}$ & 1.974993 & 0.2998 & & \\
\hline & $a_{2}$ & 0.832715 & 0.2316 & & \\
\hline \multirow[t]{2}{*}{6} & ao & 0.00004 & 0.000016 & 0.025 & 0.770 \\
\hline & $a_{1}$ & 1.57377 & 0.1164 & & \\
\hline \multirow[t]{3}{*}{7} & ao & 0.000007 & 0.000003 & 0.026 & 0.845 \\
\hline & $a_{1}$ & 2.57172 & 0.1850 & & \\
\hline & $a_{2}$ & 0.310612 & 0.1920 & & \\
\hline \multirow[t]{3}{*}{8} & ao & 0.000027 & 0.000011 & 0.012 & 0.859 \\
\hline & $a_{1}$ & 1.857894 & 0.1825 & & \\
\hline & $a_{2}$ & 0.544918 & 0.2174 & & \\
\hline \multirow[t]{2}{*}{9} & ao & 0.000047 & 0.000025 & 0.035 & 0.645 \\
\hline & $a_{1}$ & 1.570357 & 0.1486 & & \\
\hline \multirow[t]{3}{*}{10} & ao & 0.000009 & 0.000003 & 0.018 & 0.833 \\
\hline & $a_{1}$ & 2.332987 & 0.1451 & & \\
\hline & $a_{2}$ & 0.41663 & 0.1161 & & \\
\hline \multirow[t]{3}{*}{11} & ao & 0.000011 & 0.000005 & 0.024 & 0.849 \\
\hline & $a_{1}$ & 2.308282 & 0.1666 & & \\
\hline & $a_{2}$ & 0.368689 & 0.1539 & & \\
\hline \multirow[t]{3}{*}{12} & ao & 0.000053 & 0.000042 & 0.016 & 0.730 \\
\hline & $a_{1}$ & 1.731934 & 0.3564 & & \\
\hline & $a_{2}$ & 0.529313 & 0.2683 & & \\
\hline \multirow[t]{3}{*}{170} & ao & 0.000008 & 0.000003 & 0.026 & 0.870 \\
\hline & $a_{1}$ & 2.358227 & 0.1976 & & \\
\hline & $a_{2}$ & 0.539605 & 0.2360 & & \\
\hline \multirow[t]{3}{*}{172} & ao & 0.000069 & 0.000029 & 0.033 & 0.788 \\
\hline & $a_{1}$ & 1.724895 & 0.1900 & & \\
\hline & $a_{2}$ & 0.715981 & 0.1761 & & \\
\hline
\end{tabular}

Sp = Código de identificación de la especie, Parm = Parámetros del modelo, EStd = Error estándar; 1 = Pinus cooperi, $2=P$. durangensis, $3=P$. engelmannii, $4=P$. leiophylla, $5=P$. herrerae, $6=P$. teocote, $7=P$. lumholtzii, $8=P$. ayacahuite, $9=P$. oocarpa, $10=P$. douglasiana, $11=P$. michoacana, $12=P$. maximinoi, $170=$ Quercus spp, $172=$ Q. durifolia . 


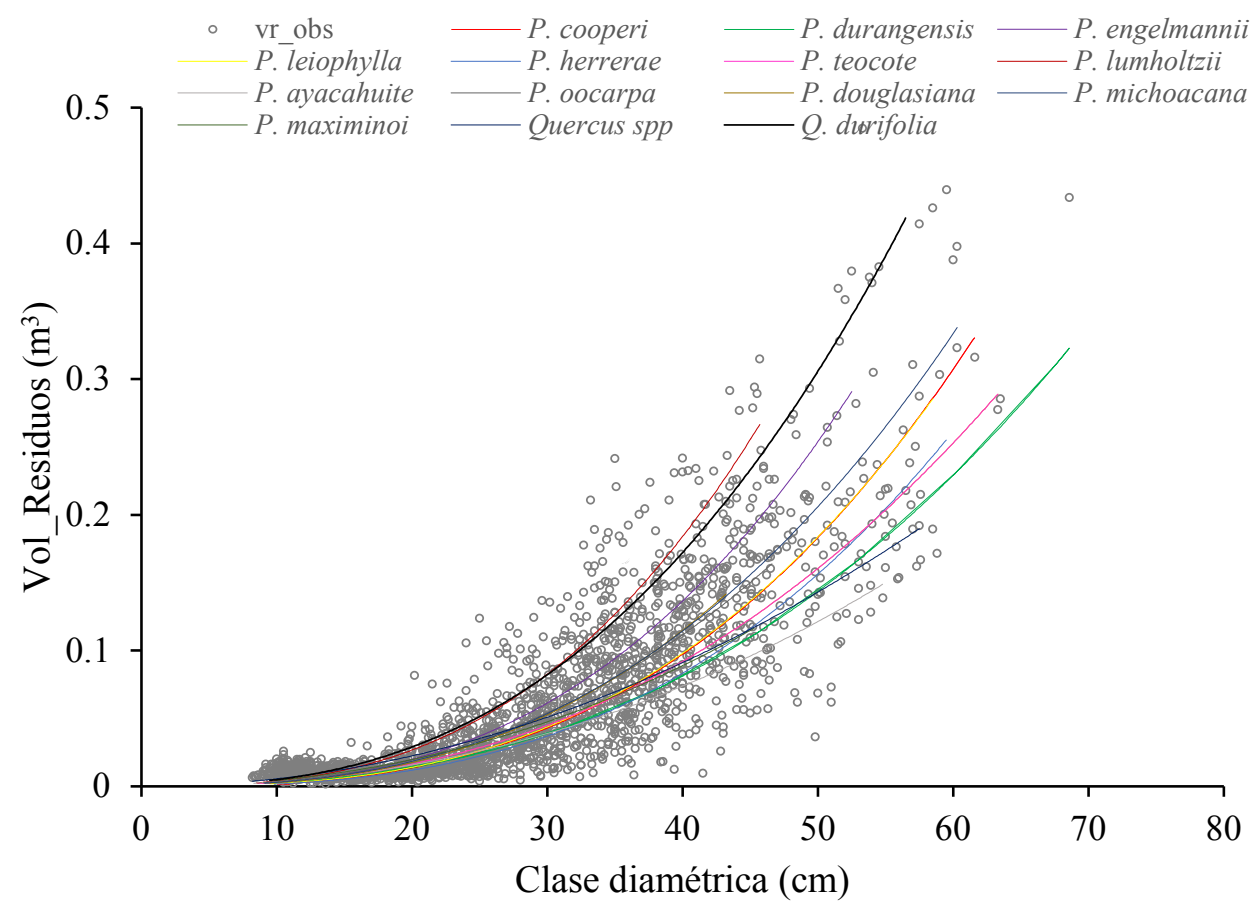

FIGURA 3. Curvas de volumen de residuos forestales por categoría diamétrica por especie, sobrepuestas a los valores observados de residuos (vr_obs).

\section{Generación de electricidad y desplazamiento de carbono}

En las tablas 6 y 7 se presentan los valores de energía eléctrica ( $G W$ h) que se puede producir a partir de los residuos de pino y encino, considerando una eficiencia en la generación de energía de 40\%. La capacidad promedio de generación de electricidad considerando los residuos de pino (Tabla 6) y de encino (Tabla 7) fue de 65.6 GW h año-1 para un ciclo de corta de 10 años.

\section{DISCUSIÓN}

\section{Modelo predictivo de residuos a escala de árbol individual}

En este estudio se consideró como residuo el volumen proveniente de puntas y ramas de los árboles (sin considerar el tocón), clasificación de residuos que ha sido utilizada en otros estudios (Gan y Smith, 2006). Las ecuaciones generadas para estimar el volumen de residuos se basan en un modelo general de tipo alométrico que considera como variables explicativas al diámetro normal y el diámetro de copa (ec. 2). El hecho de que se hayan obtenido buenos ajustes con este modelo se debe, en gran medida, a la inclusión de la dimensión de la copa, en la cual se concentra la mayor cantidad de residuos. Peltola et al. (2011) utilizaron un modelo de tres variables que incluye la longitud de la copa del árbol, cuya inclusión también significó una ganancia en los ajustes del modelo y mejoró las estimaciones de la cantidad de residuos de la copa. De acuerdo con Fassola et al. (2008), incorporar otras variables además del diámetro normal en un modelo de este tipo, permite tener criterios de selección con fundamentos biológicos, de tal forma que los modelos permiten incorporar información sobre la acumulación de tejidos en los árboles, la arquitectura de la copa y consecuentemente la estructura de las ramas, principal fuente de residuos forestales. 
TABLA 3. Prueba de F generalizada

\begin{tabular}{|c|c|c|c|c|c|c|}
\hline$S p$ & SSE (R) & $d f_{R}$ & SSE (C) & $d f_{c}$ & $F^{*}$ & $P r>|F|$ \\
\hline $1-2$ & 0.4814 & 358 & 0.4577 & 357 & 18.48 & 0.0001 \\
\hline $1-3$ & 0.5164 & 330 & 0.4062 & 329 & 89.25 & 0.0001 \\
\hline $1-4$ & 0.4324 & 338 & 0.4213 & 335 & 2.94 & 0.0331 \\
\hline $1-5$ & 0.4332 & 312 & 0.4247 & 309 & 2.06 & 0.1053 \\
\hline $1-6$ & 0.3965 & 341 & 0.3908 & 340 & 4.95 & 0.02 \\
\hline $1-7$ & 0.4292 & 271 & 0.3731 & 270 & 40.59 & 0.0001 \\
\hline $1-8$ & 0.3196 & 270 & 0.3113 & 269 & 7.12 & 0.007 \\
\hline $1-9$ & 0.4900 & 324 & 0.4624 & 323 & 19.27 & 0.0001 \\
\hline $1-10$ & 0.3702 & 381 & 0.3580 & 380 & 12.94 & 0.0003 \\
\hline $1-11$ & 0.4003 & 305 & 0.3821 & 304 & 14.47 & 0.0001 \\
\hline $1-12$ & 0.3170 & 251 & 0.3148 & 248 & 0.57 & 0.6301 \\
\hline $2-3$ & 0.4681 & 357 & 0.2681 & 356 & 265.57 & 0.0001 \\
\hline $2-4$ & 0.3076 & 365 & 0.2863 & 364 & 27.08 & 0.0001 \\
\hline $2-5$ & 0.3017 & 339 & 0.2888 & 337 & 7.52 & 0.0006 \\
\hline $2-6$ & 0.2522 & 368 & 0.2414 & 366 & 8.18 & 0.0003 \\
\hline $2-7$ & 0.4009 & 298 & 0.2288 & 297 & 223.39 & 0.0001 \\
\hline $2-8$ & 0.1766 & 297 & 0.1734 & 294 & 1.80 & 0.1456 \\
\hline $2-9$ & 0.3877 & 351 & 0.3210 & 350 & 72.72 & 0.0001 \\
\hline $2-10$ & 0.2589 & 408 & 0.2198 & 407 & 72.40 & 0.0001 \\
\hline $2-11$ & 0.3010 & 332 & 0.2410 & 331 & 82.40 & 0.0001 \\
\hline $2-12$ & 0.1862 & 278 & 0.1802 & 277 & 9.22 & 0.0026 \\
\hline $3-4$ & 0.3356 & 337 & 0.2392 & 336 & 135.41 & 0.0001 \\
\hline $3-5$ & 0.3978 & 311 & 0.2396 & 309 & 102.01 & 0.0001 \\
\hline $3-6$ & 0.3099 & 340 & 0.1970 & 339 & 194.27 & 0.0001 \\
\hline $3-7$ & 0.2083 & 270 & 0.1806 & 269 & 41.25 & 0.0001 \\
\hline $3-8$ & 0.2194 & 269 & 0.1244 & 268 & 204.66 & 0.0001 \\
\hline $3-9$ & 0.2742 & 323 & 0.2638 & 322 & 12.69 & 0.0004 \\
\hline $3-10$ & 0.1838 & 380 & 0.1715 & 379 & 27.18 & 0.0001 \\
\hline $3-11$ & 0.2140 & 304 & 0.1882 & 303 & 41.53 & 0.0001 \\
\hline $3-12$ & 0.1434 & 250 & 0.1295 & 249 & 26.72 & 0.0001 \\
\hline $4-5$ & 0.2784 & 319 & 0.2574 & 317 & 12.93 & 0.0001 \\
\hline $4-6$ & 0.2251 & 348 & 0.2205 & 347 & 13.50 & 0.0001 \\
\hline $4-7$ & 0.3280 & 278 & 0.1979 & 277 & 7.239 & 0.0074 \\
\hline $4-8$ & 0.1572 & 277 & 0.1418 & 274 & 9.91 & 0.0001 \\
\hline $4-9$ & 0.3118 & 331 & 0.2851 & 330 & 30.90 & 0.0001 \\
\hline $4-10$ & 0.2074 & 388 & 0.1902 & 387 & 34.99 & 0.0001 \\
\hline $4-11$ & 0.2262 & 312 & 0.2104 & 311 & 23.35 & 0.0001 \\
\hline $4-12$ & 0.1506 & 258 & 0.1472 & 255 & 1.96 & 0.1199 \\
\hline $5-6$ & 0.2371 & 322 & 0.2256 & 320 & 8.15 & 0.0001 \\
\hline $5-7$ & 0.3608 & 252 & 0.2153 & 251 & 169.62 & 0.0001 \\
\hline $5-8$ & 0.1507 & 251 & 0.1452 & 248 & 3.13 & 0.0262 \\
\hline $5-9$ & 0.3582 & 305 & 0.2828 & 303 & 40.39 & 0.0001 \\
\hline $5-10$ & 0.2248 & 362 & 0.1915 & 360 & 31.30 & 0.0001 \\
\hline $5-11$ & 0.2649 & 286 & 0.2268 & 285 & 47.87 & 0.0001 \\
\hline $5-12$ & 0.1596 & 232 & 0.1550 & 231 & 6.85 & 0.0094 \\
\hline $6-7$ & 0.2774 & 281 & 0.1540 & 280 & 224.36 & 0.0001 \\
\hline $6-8$ & 0.1030 & 280 & 0.0971 & 279 & 16.95 & 0.0001 \\
\hline $6-9$ & 0.2700 & 334 & 0.2260 & 333 & 64.83 & 0.0001 \\
\hline $6-10$ & 0.1654 & 391 & 0.1480 & 389 & 22.86 & 0.0001 \\
\hline $6-11$ & 0.1921 & 315 & 0.1609 & 314 & 60.88 & 0.0001 \\
\hline $6-12$ & 0.1036 & 261 & 0.1023 & 258 & 1.09 & 0.3526 \\
\hline $7-8$ & 0.2162 & 210 & 0.0856 & 209 & 318.87 & 0.0001 \\
\hline $7-9$ & 0.2691 & 264 & 0.2193 & 261 & 19.75 & 0.0001 \\
\hline $7-10$ & 0.1951 & 321 & 0.1309 & 319 & 78.22 & 0.0001 \\
\hline $7-11$ & 0.2120 & 245 & 0.1485 & 244 & 104.33 & 0.0001 \\
\hline $7-12$ & 0.1399 & 191 & 0.0908 & 190 & 102.74 & 0.0001 \\
\hline $8-9$ & 0.2162 & 263 & 0.1657 & 262 & 79.84 & 0.0001 \\
\hline $8-10$ & 0.1035 & 320 & 0.0793 & 319 & 97.34 & 0.0001 \\
\hline $8-11$ & 0.1327 & 244 & 0.0937 & 243 & 101.14 & 0.0001 \\
\hline $8-13$ & 0.0392 & 190 & 0.0353 & 189 & 20.88 & 0.0001 \\
\hline $9-10$ & 0.2202 & 374 & 0.2140 & 372 & 5.38 & 0.0049 \\
\hline $9-11$ & 0.2308 & 298 & 0.2280 & 295 & 1.20 & 0.3071 \\
\hline $9-12$ & 0.1784 & 244 & 0.1701 & 241 & 3.91 & 0.0092 \\
\hline $10-11$ & 0.1435 & 355 & 0.1405 & 353 & 3.76 & 0.0240 \\
\hline $10-12$ & 0.0846 & 301 & 0.0830 & 300 & 5.78 & 0.0167 \\
\hline $11-12$ & 0.1021 & 225 & 0.0992 & 224 & 6.54 & 0.0111 \\
\hline $170-172$ & 05.0006 & 309 & 4.9293 & 306 & 1.475 & 0.2212 \\
\hline
\end{tabular}

Un valor de $\mathrm{P}<0.05$ indica que los modelos son diferentes. $\mathrm{Sp}$ = especies comparadas (códigos definidos en tabla 2), SSE (R) = suma de cuadrados del error del modelo reducido, $d f_{R}=$ grados de libertad del modelo reducido, SSE (C) = suma de cuadrados del error del modelo completo, $d f_{c}=$ grados de libertad del modelo completo, $F^{*}=$ valor calculado de $F$ y $\operatorname{Pr}|F|=$ probabilidad calculada. 
TABLA 4. Parámetros estimados y estadísticos de bondad de ajuste del modelo reducido.

\begin{tabular}{|c|c|c|c|c|c|c|c|}
\hline$S p$ & Parm & Estimador & EStd & Valor-t & $P>|t|$ & REMC & $R_{a d j}^{2}$ \\
\hline \multirow[t]{3}{*}{$1-5$} & aо & 0.000002 & 0.000001 & 1.95 & 0.05 & 0.0373 & 0.79 \\
\hline & $a_{1}$ & 2.350397 & 0.1685 & 13.95 & $<.0001$ & & \\
\hline & $a_{2}$ & 0.891439 & 0.1323 & 6.74 & $<.0001$ & & \\
\hline \multirow[t]{3}{*}{$1-12$} & ao & 0.000005 & 0.000003 & 2.00 & 0.0466 & 0.0355 & 0.80 \\
\hline & $a_{1}$ & 2.227561 & 0.1663 & 13.39 & $<.0001$ & & \\
\hline & $a_{2}$ & 0.763729 & 0.1373 & 5.56 & $<.0001$ & & \\
\hline \multirow[t]{3}{*}{$2-8$} & ao & 0.000002 & 0.000010 & 2.67 & 0.0080 & 0.0244 & 0.86 \\
\hline & $a_{1}$ & 2.610795 & 0.1364 & 19.15 & $<.0001$ & & \\
\hline & $a_{2}$ & 0.362278 & 0.1191 & 3.04 & 0.0026 & & \\
\hline \multirow[t]{3}{*}{$4-12$} & ao & 0.000002 & 0.000009 & 2.30 & 0.0221 & 0.0242 & 0.80 \\
\hline & $a_{1}$ & 2.798942 & 0.1520 & 18.42 & $<.0001$ & & \\
\hline & $a_{2}$ & 0.190213 & 0.1171 & 1.62 & 0.1056 & & \\
\hline \multirow[t]{3}{*}{$5-8$} & ao & 0.000003 & 0.000002 & 2.00 & 0.0462 & 0.0245 & 0.81 \\
\hline & $a_{1}$ & -0.20324 & 0.1841 & 11.39 & $<.0001$ & & \\
\hline & $a_{2}$ & 0.559941 & 0.1609 & 7.24 & $<.0001$ & & \\
\hline \multirow[t]{3}{*}{$6-12$} & ao & 0.000019 & 0.000005 & 3.45 & $<.0007$ & 0.0199 & 0.81 \\
\hline & $a_{1}$ & 2.207211 & 0.0993 & 22.22 & $<.0001$ & & \\
\hline & $a_{2}$ & 0.179616 & 0.0982 & 1.83 & $<.0686$ & & \\
\hline \multirow[t]{3}{*}{$9-11$} & ao & 0.000012 & 0.000005 & 2.58 & 0.0104 & 0.0278 & 0.79 \\
\hline & $a_{1}$ & 2.420499 & 0.1257 & 19.25 & $<.0001$ & & \\
\hline & $a_{2}$ & 0.161674 & 0.0903 & 1.79 & 0.0743 & & \\
\hline \multirow[t]{3}{*}{$170-172$} & ao & 0.000023 & 0.000007 & 3.42 & 0.0007 & 0.0311 & 0.82 \\
\hline & $a_{1}$ & 1.922 & 0.1021 & 18.83 & $<.0001$ & & \\
\hline & $\mathrm{a}_{2}$ & 0.872872 & 0.1055 & 8.27 & $<.0001$ & & \\
\hline
\end{tabular}

Sp = especies comparadas (códigos definidos en tabla 2), Parm = parámetros del modelo, EStd = error estándar, P> $|\mathrm{t}|=$ probabilidad calculada

TABLA 5. Parámetros estimados y estadísticos de bondad de ajuste por grupo de especies.

\begin{tabular}{lcllllll}
\hline Grupo & Parm & Estimador & EStd & Valor-t & $P>|t|$ & $R E M C$ & $R_{\text {adj }}^{2}$ \\
\hline Pinus & ao & 0.000012 & 0.000002 & 6.17 & $<0.0001$ & 0.0322 & 0.749 \\
& a1 & 2.512722 & 0.0411 & 61.11 & $<0.0001$ & & \\
& a 2 & -0.07478 & 0.0242 & -3.09 & $<0.0020$ & & \\
\hline Quercus & ao & 0.000023 & 0.000007 & 3.42 & 0.0007 & 0.0311 & 0.822 \\
& a1 & 1.922 & 0.1021 & 18.83 & $<0.0001$ & & \\
& a2 & 0.872872 & 0.1055 & 8.27 & $<0.0001$ & & \\
\hline
\end{tabular}

Grupo = Grupo de especies, Parm = Parámetros del modelo, EStd = Error estándar, $\mathrm{P}>|\mathrm{t}|=$ Probabilidad calculada 
TABLA 6. Conversión del volumen de residuos de pino a energía eléctrica.

\begin{tabular}{cccccc}
\hline Año & $\begin{array}{c}\text { Superficie } \\
(\mathrm{ha})\end{array}$ & $\begin{array}{c}\mathrm{V} r \\
\left(\mathrm{~m}^{3}\right)\end{array}$ & $\begin{array}{c}\text { Biomasa } \\
(\mathrm{Mg})\end{array}$ & $\begin{array}{c}\text { Energía } \\
(\mathrm{MWh})\end{array}$ & $\begin{array}{c}\text { Dióxido de } \\
\text { carbono } \\
\text { equivalente } \\
(\mathrm{LCO} e)\end{array}$ \\
\hline 1 & 9357 & 50150 & 23069 & 48.9 & 12457 \\
2 & 8802 & 41634 & 19152 & 40.6 & 10342 \\
3 & 8663 & 41865 & 19258 & 40.8 & 10399 \\
4 & 8846 & 41052 & 18884 & 40.0 & 10197 \\
5 & 8105 & 39895 & 18352 & 38.9 & 9910 \\
6 & 8553 & 39911 & 18359 & 38.9 & 9914 \\
7 & 8216 & 40232 & 18507 & 39.2 & 9994 \\
8 & 8565 & 40209 & 18496 & 39.2 & 9988 \\
9 & 8037 & 39511 & 18175 & 38.5 & 9815 \\
10 & 8281 & 40048 & 18422 & 39.1 & 9948 \\
\hline Total & 85425 & 414507 & 190673 & 404 & 102964 \\
\hline
\end{tabular}

$V_{r}=V_{\text {ramas }}+V_{\text {punta }}$

TABLA 7. Conversión del volumen de residuos de encino a energía eléctrica.

\begin{tabular}{cccccc}
\hline Año & $\begin{array}{c}\text { Superficie } \\
(\mathrm{ha})\end{array}$ & $\begin{array}{c}\mathrm{Vr} \\
\left(\mathrm{m}^{3}\right)\end{array}$ & $\begin{array}{c}\text { Biomasa } \\
(\mathrm{Mg})\end{array}$ & $\begin{array}{c}\text { Energía } \\
(\mathrm{MWh})\end{array}$ & $\begin{array}{c}\text { Dióxido de carbono } \\
\text { equivalente } \\
(\mathrm{LCO}) \mathrm{C}\end{array}$ \\
\hline 1 & 2900 & 15545 & 9949 & 21.1 & 5372 \\
2 & 3608 & 17067 & 10923 & 23.2 & 5898 \\
3 & 3187 & 15400 & 9856 & 20.9 & 5322 \\
4 & 3684 & 17097 & 10942 & 23.2 & 5909 \\
5 & 3432 & 16893 & 10811 & 22.9 & 5838 \\
6 & 4618 & 21546 & 13789 & 29.2 & 7446 \\
7 & 4317 & 21140 & 13530 & 28.7 & 7306 \\
8 & 4709 & 22105 & 14147 & 30.0 & 7639 \\
9 & 3717 & 18276 & 11696 & 24.8 & 6316 \\
10 & 4225 & 20432 & 13076 & 27.7 & 7061 \\
\hline Total & 38397 & 185500 & 118720 & 252 & 64109 \\
\hline
\end{tabular}


Los resultados obtenidos ajustando una ecuación por especie son considerablemente buenos (Tabla 2), con valores de REMC que oscilaron entre $0.012 \mathrm{~m}^{3}(P$. ayacahuite) y $0.0427 \mathrm{~m}^{3}$ (P. cooper $)$, es decir, entre $5.52 \mathrm{~kg}$ y $19.64 \mathrm{~kg}$ de biomasa, respectivamente, valores de REMC cercanos, e incluso más bajos, a los encontrados por Bouriaud et al. (2013) (17.8 kg de biomasa para Picea abies y $19 \mathrm{~kg}$ para Fagus sylvatica), obtenidos del ajuste de un modelo que incluye el diámetro normal y la altura total como variables independientes. Por otra parte, los valores de $\mathrm{R}_{\text {adj }}^{2}$ fueron aceptables (desde 0.645 para P. oocarpa, hasta 0.878 para P. engelmannii). Los valores más bajos de $\mathrm{R}_{\text {adj }}^{2}$ se deben, en parte, a la gran dispersión que presenta el volumen de residuos para una misma categoría diamétrica por especie (Fig. 2); además, la arquitectura y forma de la copa está altamente influenciada por la densidad de la masa, variable que no fue considerada en este estudio.

En Oaxaca, Rodríguez-Ortiz et al. (2012) ajustaron varios modelos para estimar la biomasa aérea en plantaciones de pino (Pinus patula Schiede ex Schltdl. \& Cham. y P. taeda), siendo el de mejor ajuste el mismo que se empleó en este trabajo, obteniendo valores de $\mathrm{R}_{\text {adj }}^{2}$ para la estimación de biomasa en ramas sin acículas de 0.92, considerando como variables dependientes el diámetro medido al inicio de la copa viva y el área de copa. Esto coincide también con lo señalado por Jonckheere, Muys y Copping (2005) para P. sylvestris $\left(\mathrm{R}_{\mathrm{adj}}^{2}=0.9\right)$.

\section{Comparación del volumen de residuos entre especies}

La comparación entre pares de especies de un mismo género arrojó resultados similares a los obtenidos en otros estudios. Simental-Cano et al. (2016) compararon 36 combinaciones pareadas de especies de pino y tres pares de especies de encino, y en todos los casos, excepto para el par Q. sideroxyla-Q. rugosa, se rechazó la hipótesis nula, por lo que recomiendan emplear una ecuación diferente para cada especie. Corral-Rivas, Diéguez-Aranda, Corral-Rivas y Castedo (2007) compararon el ajuste de la función compatible de ahusamiento-volumen de Fang et al. (2000) para todos las posibles combinaciones pareadas de P. cooperi, P. durangensis, P. engelmannii, P. leiophylla y P. teocote, y encontraron diferencias para todos los casos entre el modelo completo y el modelo reducido. Finalmente, Corral-Rivas, Álvarez-González, Ruíz y von Gadow (2004) también hallaron diferencias significativas en el crecimiento en altura dominante al comparar todas las posibles combinaciones pareadas de cinco de las especies del género Pinus analizadas en este estudio.

Desde el punto de vista de la reducción del error, en la mayoría de los casos no hay ganancia significativa al utilizar una misma ecuación para dos especies, por lo que no se justifica agruparlas, excepto los pares de especies de Pinus 1-5, 1-12, 2-8, 4-12, 5-8, 6-12 y 9-11. El mismo caso se presentó para Quercus durifolia (172) y el grupo Quercus spp (Q. rugosa, Q. fulva, Q. sideroxila y Q. crassifolia) donde los mejores resultados de REMC y $\mathrm{R}_{\text {adj }}^{2}$ se obtuvieron ajustando un modelo individual (Tabla 1); sin embargo, al realizar el ajuste con variables dummy (modelo completo) y ajustes globales combinando las especies (modelo reducido) no se encontró ganancia estadística al emplear uno u otro modelo $(\operatorname{Pr}|F|=0.2212)$ (Tabla 3), motivo por el cual el volumen de residuos podría estimarse con una misma ecuación para todas las especies de Quercus. De igual forma, al ajustar un único modelo a escala de género, es decir, para todas las especies de Pinus juntas, así como para todas las de Quercus, se obtuvieron estadísticos de bondad de ajuste aceptables. Por lo tanto, cuando los objetivos de la cuantificación de residuos no requieran de información a escala de especie, se puede utilizar una única ecuación a escala de género.

\section{Estimación de residuos forestales a escala regional}

Los valores obtenidos de cantidad residuos forestales provenientes de las actividades de manejo son consistentes con los publicados para el área de estudio y para otras partes del mundo. La cantidad de biomasa recuperable estimada en este estudio asciende a $30940 \mathrm{Mg}$ año-1 , cifra similar a los $28579 \mathrm{Mg}$ año- ${ }^{-1}$ de biomasa obtenida por Pérez-Verdin et al. (2012) para los bosques de la región de El Salto, 
Durango. Los valores promedio de disponibilidad de biomasa fueron $2.23 \mathrm{Mg} \mathrm{ha}^{-1}$ y $3.1 \mathrm{Mg} \mathrm{ha}^{-1}$ para Pinus y Quercus, respectivamente, superiores a los obtenidos por Peltola et al. (2011), quienes a partir de ecuaciones de residuos y datos de inventario forestal, estimaron una disponibilidad de residuos de $1.2 \mathrm{Mg} \mathrm{ha}^{-1}$ y $0.6 \mathrm{Mg} \mathrm{ha}^{-1}$ para Picea abies en el este y en el centro de Finlandia, respectivamente.

Existen pocos trabajos que han evaluado la cantidad óptima que debe permanecer en el suelo para mitigar la pérdida de nutrientes al realizar la cosecha forestal. En este estudio se consideró como cantidad mínima para recuperación de nutrientes $10 \%$ del volumen total disponible de residuos, que equivale a $0.47 \mathrm{Mg} \mathrm{ha}^{-1}$, lo cual es congruente a lo estimado por Borjesson (2000), quien sugiere que la cantidad mínima de residuos que se requiere para mantener la fertilidad del suelo puede variar entre 0.8 $\mathrm{Mg} \mathrm{ha}{ }^{-1}$ y $2.2 \mathrm{Mg} \mathrm{ha}^{-1}$ por periodo rotacional, dependiendo de las condiciones particulares de la zona.

\section{Generación de electricidad y desplazamiento de carbono}

En la región de El Salto, Durango, existen 19200 usuarios registrados (casa habitación, comercial e industrial) cuyo consumo asciende a $9.04 \mathrm{GW}$ h por bimestre, es decir, la demanda de energía eléctrica asciende a $54.2 \mathrm{GW} \mathrm{h}$ año ${ }^{-1}$, la cual se podría cubrir con los $66.5 \mathrm{GW}$ h año-1 que potencialmente se podrían producir con un sistema de generación a base de biomasa. Estos resultados son congruentes con los mencionados por Gan y Smith (2006) para algunas regiones de Estados Unidos; por ejemplo, para la región del estado de Iowa señalan una disponibilidad de $29000 \mathrm{Mg}$ de residuos forestales secos con una capacidad de generación de electricidad de 55 MW h (con una eficiencia de $35 \%$ ), lo que equivale a $61.5 \mathrm{MW}$ h para una eficiencia de $40 \%$, similar a lo obtenido en este estudio. Por otra parte, los resultados encontrados por Simangunson et al. (2017) para la misma cantidad de residuos disponibles estimados en este estudio (30 $940 \mathrm{Mg}$ año-1) equivaldría a 63.11 GW h, lo cual demuestra la robustez de la metodología utilizada. $\mathrm{Si}$ los residuos forestales recuperables se usaran para la generación de energía eléctrica, la reducción de emisiones promedio en un ciclo de corta de 10 años sería 16707 tCO $\mathrm{CO}_{2}$ año ${ }^{-1}$ (Tablas 6 y 7), resultado similar al obtenido por Gan y Smith (2006), quienes señalan que la utilización de 29000 t de residuos para generar electricidad significa una reducción de emisiones de $15660 \mathrm{tCO}_{2} \mathrm{e}$ año-1.

\section{CONCLUSIONES}

Este estudio evaluó la disponibilidad de residuos de la cosecha forestal a escala de árbol individual y a escala regional en Pueblo Nuevo, Durango. El uso de un modelo individual para cada especie proporcionó los mejores ajustes para la mayoría de las especies; solo en 9 de los 67 pares de especies analizados para evaluar diferencias en el volumen de residuos no fue posible rechazar la hipótesis de un modelo único. Sin embargo, cuando los objetivos de la cuantificación de residuos no requieran de información a escala de especie, se puede utilizar una única ecuación a escala de género. La cantidad estimada de residuos recuperables asciende a casi $31000 \mathrm{Mg}$ de biomasa por año. Si estos residuos son usados para generación de electricidad se pueden producir hasta $65.6 \mathrm{GW} \mathrm{h}$ año-1, lo que equivaldría a una reducción de emisiones de $16707 \mathrm{tCO}_{2} \mathrm{e}$ por año. Se recomienda complementar los resultados de este estudio con análisis adicionales sobre los costos de transporte de los residuos considerando distintas opciones de acopio de la materia prima, es decir, considerar la distribución espacial de los residuos y la accesibilidad a los mismos, así como distintas tecnologías de generación de electricidad a partir de biomasa forestal y sus respectivos costos de producción.

\section{RECONOCIMIENTOS}

Este estudio fue financiado por el Consejo de Ciencia y Tecnología del Estado de Durango (Cocyted), a través del proyecto: "Estimación del potencial de los bosques de Durango para la mitigación del cambio climático" (clave: FOMIX-DGO-2011-C01-165681). 


\section{REFERENCIAS}

Bates, D. M., \& Watts, D. G. (1988). Nonlinear Regression Analysis and Its Applications. New York: John Wiley and Sons.

Borjesson, P. (2000). Economic valuation of the environmental impact of logging residue recovery and nutrient compensation. Biomass and Bioenergy, 19(3), 137-152. doi:10.1016/S0961-9534(00)00028-3

Bouriaud, O., Stefan, G., \& Flocea, M. (2013). Predictive models of forest logging residues in Romanian spruce and beech forests. Biomass and Bioenergy, 54, 59-66. doi:10.1016/j.biombioe.2013.03.022

Corral-Rivas, J. J., Álvarez-González, J. G., Ruíz, A. D., \& v. Gadow, K. (2004). Compatible height and site index models for five pine species in El Salto, Durango (Mexico). Forest Ecology and Management, 201(2-3), 145-160. doi:10.1016/j.foreco.2004.05.060

Corral-Rivas, J. J., Diéguez-Aranda, U., Corral-Rivas, S., \& Castedo, D. F. (2007). A merchantable volume system for major pine species in El Salto, Durango (Mexico). Forest Ecology and Management, 238(1-3), 118-129. doi:10.1016/j.foreco.2006.09.074

Draper, N., \& Smith, H. (1981). Applied Regression Analysis (2a ed.) USA: John Wiley \& Sons.

Evans, A., Strezov, V., \& Evans, T. J. (2010). Sustainability considerations for electricity generation from biomass. Renewable and Sustainable Energy Reviews, 14(5), 1419-1427. doi:10.1016/i.rser.2010.01.010

Fang, Z., \& Bailey, R. L. (2001). Nonlinear mixed effects modeling for slash pine dominant height growth following intensive silvicultural treatments. Forest Science, 47(3), 287300. doi:10.1093/forestscience/47.3.287

Fassola, H., Crechi, E., Barth, S., Keller, A., Pinazo, M., Martiarena, R., Von Wallis A., \& Figueredo, I. (2008). Relación entre la biomasa aérea y la sección transversal en la base de copa viva de Pinus taeda L. en el norte de la provincia de Misiones, Argentina. XIII Jornadas Técnicas Forestales y Ambientales. Facultad de Ciencias Forestales, UNAM-EEA Montecarlo, INTA. doi:10.13140/2.1.4631.8889

Ferranti, F. (2014). Energy wood: A challenge for European forests potentials, environmental implications, policy integration and related conflicts. European Forest Institute. Recuperado de http://www.efi.int/files/attachments/publications/efi_tr _95_ferranti_2014.pdf
Gan, J., \& Smith, C. T. (2006). A comparative analysis of woody biomass and coal for electricity generation under various $\mathrm{CO}_{2}$ emission reductions and taxes. Biomass and Bioenergy, 30(4), 296-303. doi:10.1016/j.biombioe.2005.07.006

Hamelinck, C. N., Van Hooijdonk, G., \& Faaij, A. P. (2005). Ethanol from lignocellulosic biomass: techno-economic performance in short-, middle- and long-term. Biomass and Bioenergy, 28(4), 384-410. doi:10.1016/j.biombioe.2004.09.002

Hammerschlag, R. (2006). Ethanol's energy return on investment: a survey of the literature 1990-present. Environmental Science and Technology, 40(6), 1744-50. doi:10.1021/es052024h

Harvey, A. C. (1976). Estimating regression models with multiplicative heteroscedasticity. Econometrica, 44(3), 461465. doi:10.2307/1913974

Hauglin, M. Gobakken, T. Lien, V. Bollandsas, O. M., \& Naesset, E. (2012). Estimating potential logging residues in a boreal forest by airborne laser scanning. Biomass and Bioenergy, 36, 356-365. doi:10.1016/j.biombioe.2011.11.004

Islas, j. Manzini, F., \& Masera, O. (2007). A prospective study of bioenergy use in México. Energy, 32(12), 2306-2320. doi:10.1016/j.energy.2007.07.012

Johnson, T. M., Alatorre, C. Romo, Z., \& Liu, F. (2009). México: estudio sobre la disminución de emisiones de carbón (MEEDEC). México: Mayol Ediciones S.A. Recuperado de http://awsassets.panda.org/downloads/estudio_sobre_la _disminucion_de_emisiones_de_carbono.pdf

Jonckheere, I., Muys, B., \& Copping, P. (2005). Allometry and evaluation of in situ optical LAI determination in Scots pine: a case study in Belgium. Tree Physiology, 25(6), 723-732. doi:10.1093/treephys/25.6.723

Kennedy, M. (2008). Assessment of Potential for Emission Reduction Credits in Durango, Mexico. The Pembina Institute. p 30 .

Masera, O., Aguillón, J. Arvizu, J. Fuentes, A. Ghilardi, A. Riegelhaupt, E. Saldaña, R. Best, G. Gómez, I. Díaz, R. Gamiño, R. Berrueta, V. Islas, J. Manzini, F., \& Rodríguez, O. (2006). La bioenergía en México un catalizador del desarrollo sustentable. Comisión Nacional Forestal. 80 p. Recuperado de http://rembio.org.mx/wpcontent/uploads/2014/10/libro-blanco-bioenergia2006.pdf

Montgomery, D., \& Peck, E. (1982). Introduction to linear regression analysis. New York: John Wiley \& Sons. 
Organización de las Naciones Unidas para la Alimentación y la Agricultura [FAO] (2010). W hat woodfuels can do to mitigate climate change? Roma. Recuperado de http://www.fao.org/docrep/013/i1756e/i1756e00.htm

Peltola, S. Kilpelainen, H., \& Asikainen, A. (2011). Recovery rates of logging residue harvesting in Norway spruce (Picea abies (L.) Karsten) dominated stands. Biomass and Bioenergy, 35(4), 1545-1551. doi:10.1016/j.biombioe.2010.12.032

Pérez-Verdín, G., Navar-Chaidez, J. Grebner, D., \& SotoÁlvarez, C. (2012). Disponibilidad y costos de producción de biomasa forestal como materia prima para la producción de bioetanol. Forest systems, 21(3), 526-537. doi:10.5424/fs/2012213-02636

Pro Floresta, S. C. (2008). Estudio Regional Forestal UMAFOR 1008, Pueblo Nuevo, Durango. 397 p.

Rodríguez-Ortiz, G., De Los Santos-Posadas, H. M. GonzálezHernández, V., Aldrete, A. Gómez-Guerrero, A., \& Fierros-González, A. (2012). Modelos de biomasa aérea y foliar en una plantación de pino de rápido crecimiento en Oaxaca. Madera y Bosques, 18(1), 25-41. doi: 10.21829/myb.2012.1811116

Statistical Analysis System [SAS] (2011). SAS/ETS® 9.3 User's Guide. Cary, NC: SAS Institute Inc.

Scarlat, N. Blujdea, V., \& Dallemand, J. F. (2011). Assessment of the availability of agricultural and forest residues for bioenergy production in Romania. Biomass and Bioenergy, 35(5), 1995-2005. doi:10.1016/j.biombioe.2011.01.057

Simangusong, B. C. H., Sitanggang, V. J., Manurung, E. G. T., Rahmadi, A., Moore, G. A., Aye, L., \& Tambunan, A. H. (2017). Potential forest biomass resource as feedstock for bioenergy and its economic value in Indonesia. Forest Policy and Economics, 81, 10-17. doi.org/10.1016/j.forpol.2017.03.022

Simental-Cano, B., López-Sánchez, C. A., Wehenkel, C., VargasLarreta, B., Álvarez-González, J. G., \& Corral-Rivas, J. J.
(2016). Modelos de volumen específicos y regionales para 12 especies forestales en el estado de Durango, México. Revista Chapingo. Serie Ciencias Forestales y del Ambiente, 23(2), 155-171. doi.org/10.5154/r.rchscfa.2016.01.004

Smeets, E. M., \& Faaij, A. P. (2007). Bioenergy potentials from forestry in 2050. Climatic Change, 81(3-4), 353-390. doi:10.1007/s10584-006-9163-x

Weisberg, S. (1980). Applied Linear Regression (3a ed.), New York: John Wiley \& Sons.

World Wildlife Fund, \& Hogan, M. (2013). Sierra Madre Occidental pine-oak forests. Encyclopedia of Earth, National Council for Science and the Environment. Washington DC: Rodney L. H. Recuperado de http://eol.org/data_objects/27361823.

Wright, L., Boundy, B., Perlack, B., Davis, S., \& Saulsbury, B. (2006). Biomass energy data book (Vol. 1). US Department of Energy Publications. University of Nebraska. Publication No. 4.

Manuscrito recibido el 2 de marzo de 2017

Aceptado el 22 de febrero de 2018

Publicado el 1 de noviembre de 2018

Este documento se debe citar como:

Villela-Suárez, J. M., Aguirre-Calderón, O. A., Treviño-Garza, E. J., \& Vargas-Larreta, B. (2018). Disponibilidad de residuos forestales y su potencial para la generación de energía en los bosques templados de El Salto, Durango. Madera y Bosques, 24(3), e2431529. doi: 10.21829/myb.2018.2431529

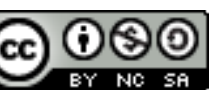

Madera y Bosques por Instituto de Ecología, A.C. se distribuye bajo una Licencia Creative Commons Atribución-NoComercialCompartirlgual 4.0 Internacional. 